\title{
Reasons to Not Believe (and Reasons to Act)
}

\author{
BLAKE ROEBER \\ University of Notre Dame
}

It's an honor to comment on Stewart Cohen's paper "Reasons to Believe and Reasons to Act." I read "How to be a Fallibilist" and "Contextualist Solutions to Epistemological Problems" as an undergraduate, and I have learned from Cohen's work since. It is consistently interesting and compelling. "Reasons to Believe and Reasons to Act" is no exception. While my comments here are mostly critical, I find many of its central ideas convincing.

In "Reasons to Believe and Reasons to Act," Cohen argues that balance of reasons accounts of rational action get the wrong results when applied to doxastic attitudes. Specifically, he argues that we get counterexamples to BALANCE (below) when we apply it to belief, disbelief, and suspension of judgement.

BALANCE: $S$ is rationally permitted to $\varphi$ just in case her reasons to $\varphi$ are at least as strong as her reasons to not $\varphi$, and she is rationally required to $\varphi$ just in case her reasons to $\varphi$ are stronger than her reasons to not $\varphi$.

According to Cohen, unless there are non-evidential reasons to withhold belief, BALANCE entails that $S$ will be rationally permitted to believe $p$ in cases where she is clearly rationally required to withhold. Specifically, it entails that $S$ will be rationally permitted to believe $p$ in cases where her evidence for $p$ equals her evidence for $\neg p$. But first, says Cohen, it seems doubtful that there are non-evidential reasons to withhold. The best reasons for thinking that there are come from Schroeder's cases involving practical interests, forthcoming evidence, and the mental resources required for forming a belief, but none of these cases provides a clear example of nonevidential reasons to withhold. And second, says Cohen, even if one of these cases does provide a clear example of non-evidential reasons to withhold, these reasons to withhold will often be inoperative. The result will be that BALANCE still entails that $S$ will be rationally permitted to believe $p$ in some cases where her evidence for $p$ equals her evidence for $\neg p$. Things get even worse for BALANCE, however, says Cohen. Even if we waive these worries, BALANCE entails that one may be rationally permitted to

* Penultimate draft. Please cite final version forthcoming in Episteme. 
take multiple doxastic attitudes toward a proposition, and it will often get the wrong result in cases where someone is simply not considering a proposition. Because BALANCE gets the right results when applied to action-or, at least, because it gets the right results when applied to relevantly analogous cases of action-Cohen concludes that there are important differences between reasons to believe and reasons to act.

I agree with Cohen that Schroeder's cases involving practical interests, forthcoming evidence, and the mental resources required for forming a belief do not provide clear examples of non-evidential reasons to withhold, and I also agree with Cohen that, even if they did, these reasons would often be inoperative. I am not yet convinced, however, that there are important differences between reasons to believe and reasons to act. In these comments, I will try to resist each of Cohen's objections to BALANCE. First, I will argue that BALANCE does not entail that $S$ will be rationally permitted to believe $p$ in some cases where her evidence for $p$ equals her evidence for $\neg p$. Second, I will argue that there are cases where more than one doxastic attitude seems rationally permissible. And third, I will argue that BALANCE gets the right results in cases where someone is not considering a proposition, unless it gets the wrong results in analogous cases involving action. I will conclude by presenting a tempting positive argument for the conclusion that there are important differences between reasons to believe and reasons to act. As I will argue, the central premise of this argument-what people often call 'transparency'—can be doubted.

\section{Reasons to Withhold}

Believing $\neg p$ is not the only way to not believe $p$. As Cohen, Schroeder (2012), and others point out, I might withhold with respect to $p$. A natural thought is that, if my evidence for $p$ equals my evidence for $\neg p$, then I have a decisive reason to not believe $p$, since this fact gives me a decisive reason to withhold with respect to $p$. With this in mind, consider the conjunction of BALANCE and EQ, below.

(EQ) If $S$ 's evidence for $p$ equals her evidence for $\neg p$, then $S$ 's reasons to not believe $p$ are stronger than her reasons to believe $p$.

According to BALANCE, if the consequent of EQ is true-if $S$ 's reasons to not believe $p$ are stronger than her reasons to believe $p$ - then $S$ is rationally required to not believe $p$. Thus, together, BALANCE and EQ entail that, if $S$ 's evidence for $p$ equals her evidence for $\neg p$, then $S$ is rationally required to not believe $p$. Unless the conjunction of BALANCE and EQ entails a contradiction, the conjunction of BALANCE and EQ 
therefore does not entail that $S$ will be rationally permitted to believe $p$ in cases where her evidence for $p$ equals her evidence for $\neg p$. If this conjunction does not entail that $S$ will be rationally permitted to believe $p$ in cases where her evidence for $p$ equals her evidence for $\neg p$, however, then BALANCE does not entail that $S$ will be rationally permitted to believe $p$ in cases where her evidence for $p$ equals her evidence for $\neg p$. (In general, if the conjunction of $A$ and $B$ does not entail $C$, then $A$ does not entail C.) Thus, so long as the conjunction of BALANCE and EQ does not entail a contradiction, BALANCE does not entail that $S$ will be rationally permitted to believe $p$ in cases where her evidence for $p$ equals her evidence for $\neg p$.

Cohen would say that the conjunction of BALANCE and EQ does entail a contradiction. EQ is necessarily false if false at all. If EQ is necessarily false, then the conjunction of BALANCE and EQ is also necessarily false. Since necessary falsehoods entail every proposition, the conjunction of BALANCE and EQ entails a contradiction if EQ is false. And according to Cohen, EQ is false.

\begin{abstract}
A natural assumption is that one's reasons to believe consist in one's evidence. It is important to see that, as Schroeder notes, evidence is always evidence for some proposition, i.e., evidence for $p$ or for $\neg p$. There cannot be evidence for not believing $p$ that is not evidence for $\neg p$. Thus if reasons to believe are evidence, whether one has sufficient reason to believe will depend on the relative strength of one's reasons to believe $p$ and one's reasons to believe $\neg p$. (p. 3)
\end{abstract}

On this way of thinking, EQ cannot be true, since $S$ 's evidence for $p$ equals her evidence for $\neg p$ iff her reasons to believe $p$ equal her reasons to not believe $p$. I agree with Cohen that evidence for $p$ is a reason to believe $p$, but I balk at the crucial third sentence of his argument. Where Cohen says " $t]$ here cannot be evidence for not believing that is not evidence for $\neg p$," I assume he means that there cannot be reasons for not believing $p$ that are not evidence for $\neg p$. (After all, if "evidence is always evidence for some proposition," as Cohen says, then evidence for $\neg p$ cannot be evidence for not believing $p$, since not believing $p$ isn't a proposition.) It seems false, however, that there cannot be reasons for not believing $p$ that are not evidence for $\neg p$. Consider the following argument. ${ }^{1}$

\footnotetext{
1 This argument takes its cue from Schroeder's (2012, p. 276) argument that reasons to withhold cannot be evidence. I do not draw the same conclusion as Schroeder, however. While I conclude that facts about one's evidence can be reasons to withhold that are not themselves evidence, Schroeder concludes that reasons to withhold do not even "come from" one's evidence. Since facts about one's evidence presumably "come from" one's evidence, Schroeder seems to think that facts about one's evidence cannot be reasons to withhold.
} 
Since withholding with respect to $p$ requires neither believing $p$ nor believing $\neg p, r$ is a reason to withhold with respect to $p$ only if $r$ is neither a reason to believe $p$ nor a reason to believe $\neg p$. But $r$ is evidence for $p$ only if $r$ is a reason to believe $p$, and $r$ is evidence for $\neg p$ only if $r$ is a reason to believe $\neg p$. So, $r$ is a reason to withhold with respect to $p$ only if $r$ is neither evidence for $p$ nor evidence for $\neg p$. Any reason to withhold with respect to $p$ would be a reason to not believe $p$, however. So, there are reasons to withhold with respect to $p$ only if there are reasons to not believe $p$ that are not evidence for $\neg p$. Are there reasons to withhold with respect to $p$, then? Yes. Let $p$ be some proposition that I cannot rationally believe without evidence (e.g. that it will rain tomorrow), suppose that I do have some evidence for $p$, but suppose that my evidence for $p$ is perfectly counterbalanced by equal evidence for $\neg p$, so that my credence in $p$ should be exactly 0.5 . This fact about my evidence (call it ' $f$ ) is a paradigm reason for me to withhold with respect to $p .^{2}$ But this fact is not itself evidence for or against $p$. Evidence for $p$ is evidence against $\neg p$, and vice versa. If $f$ is evidence for $p$, then $f$ is evidence against $\neg p$, in which case $f$ is not evidence for $\neg p$. Since $f$ lends equal support to both $p$ and $\neg p$ (if it lends any support to either of them), $f$ is evidence for both of them if it's evidence for either of them. Since it can't be evidence for both of them-it's evidence for one only if it's evidence against the other-it's not evidence for either of them. ${ }^{3}$ Thus, $f$ is a reason to withhold with respect to $p$ that is not evidence for $\neg p$. And since any reason to withhold with respect to $p$ is a reason to not believe $p$, it follows that there are reasons to not believe $p$ that are not evidence for $\neg p$.

Are there are non-evidential reasons to withhold, then? This depends on their nature-specifically, on whether NER1 or NER2 (below) gives us the correct account of non-evidential reasons to withhold.

(NER1) $r$ is a non-evidential reason for $S$ to withhold with respect to $p$ iff $r$ is a reason for $S$ to withhold with respect to $p$ that is neither evidence for $p$ nor evidence for $\neg p$.

(NER2) $r$ is a non-evidential reason for $S$ to withhold with respect to $p$ iff $r$ is a reason for $S$ to withhold with respect to $p$ that is neither evidence

\footnotetext{
${ }^{2}$ Here and throughout, I will use 'fact' as shorthand for 'true proposition.' If true propositions cannot be reasons, then I invite the reader to adjust my argument accordingly.

${ }^{3}$ And plausibly, it doesn't support either of them either. My evidence for $p$ supports $p$, and my evidence for $\neg p$ supports $\neg p$. Plausibly, neither $p$ nor $\neg p$ gets any additional support from $f$, the fact that my evidence for $p$ equals my evidence for $\neg p$.
} 
for $p$, nor evidence for $\neg p$, nor some fact about $S$ 's evidence for and against $p$.

If NER1 gives the correct account of non-evidential reasons to withhold, then there are non-evidential reasons to withhold, since $f$ is one of them. If NER2 gives the correct account of non-evidential reasons to withhold, however, then $f$ is not a nonevidential reason to withhold, and the fact that $f$ is a reason to withhold is not itself a reason to believe in non-evidential reasons to withhold. Cohen does not say exactly what he means by 'non-evidential reasons to withhold.' For my own part, I don't see any reason to prefer NER1 over NER2, so I'm happy to accept NER2 and say that reasons to withhold that are not themselves evidence needn't be non-evidential reasons to withhold. This way I can agree with Cohen that we lack clear examples of non-evidential reasons to withhold.

Either way, however, Cohen's first objection to BALANCE is not compelling. According to this objection, BALANCE entails that $S$ will be rationally permitted to believe $p$ in cases where her evidence for $p$ equals her evidence for $\neg p$, since nonevidential reasons to withhold don't exist. If NER1 gives the correct account of nonevidential reasons to withhold, Cohen is right about the connection between BALANCE and the existence of non-evidential reasons to withhold, but he's wrong about the existence of non-evidential reasons to withhold, since $f$ is a non-evidential reason to withhold. On the other hand, if NER2 gives the correct account of nonevidential reasons to withhold, Cohen might be right about the existence of nonevidential reasons to withhold, but he's wrong about the connection between BALANCE and the existence of non-evidential reasons to withhold-for in this case BALANCE is consistent with EQ even if non-evidential reasons to withhold don't exist. Either way, BALANCE survives Cohen's first objection. ${ }^{4}$

${ }^{4}$ After concluding that BALANCE does entail that $S$ is rationally permitted to believe $p$ if her evidence for $p$ equals her evidence for $\neg p$, Cohen also says this:

[BALANCE] could avoid this result if (implausibly) reasons to suspend always trump reasons to believe. But this would present a dilemma. Either there are always reasons to suspend or there are not. If the former, then it would never be rational to believe $p$. If the latter, then there will be cases where one has equally strong reasons to believe $p$ and to believe $\neg p$ and yet one is rationally permitted to believe $p$. (p. 7)

Cohen seems right here, but the point of the preceding argument is that BALANCE could also avoid this result if EQ is true. According to EQ, if $S$ 's evidence for $p$ equals her evidence for $\neg p$, she has a reason to not believe $p$ that trumps her reasons to believe $p$. EQ does not say that there are always reasons to suspend, however, or that reasons to suspend always trump reasons to believe. This means that, while BALANCE 


\title{
2. The Rational Permissibility of Multiple Attitudes
}

Cohen's second objection to BALANCE is that one may be rationally permitted to take multiple doxastic attitudes toward a proposition if BALANCE is true. Here is Cohen's argument.

\begin{abstract}
[BALANCE] views requirement as unique permissibility. When one is permitted, but not require to believe $p$, one is also permitted to suspend on $p$ and perhaps even to believe $\neg p$. This follows from the fact that one's reasons for adopting these attitudes are equal in strength. But on the standard view, one's evidence can support only one doxastic attitude toward $p$ —either belief, disbelief, or suspension. It is very hard to see how one's evidence could support believing $p$ while also supporting suspending on $p$, let alone how one's evidence could support believing $p$ while also supporting believing $\neg p$. (p. 8)
\end{abstract}

I agree with Cohen that BALANCE it entails that one may be rationally permitted to believe $p$ and simultaneously rationally permitted to not believe $p$, but I think this may be exactly the right result. Or, at least, I think this result is just as plausible as its analogue for action: that one may be rationally permitted to perform some action and simultaneously rationally permitted to not perform that action. I think there may be cases where one's evidence rules out disbelief, while providing equal support to belief and suspension. I will consider a case like this below. But notice first what BALANCE does and does not say. Since BALANCE is consistent with principles like EQ, it does not say that $S$ might be in a situation where believing $p$ and believing $\neg p$ are both rationally permissible. It also does not say that, when $S$ is permitted but not required to believe $p$, she is also permitted to suspend on $p$. This is because $S$ might have conclusive evidence for $p$, yet have reasons to consider $p$ that are perfectly counterbalanced by reasons to ignore $p$. (More on this below.) What BALANCE does say is simply that $S$ might be in a situation where believing $p$ and suspending on $p$ are both rationally permissible. ${ }^{5}$

Are there situations like this? I think there might be. To see why, imagine looking at an eye chart. At the top of the chart, there is a very large, perfectly clear 'E.' At the bottom, there a bunch of letters, but they look like dots. The transition from the perfectly clear ' $\mathrm{E}$ ' at the top of the chart to the blurry dots at the bottom is very

could avoid this result if EQ is true, EQ does not present us with the dilemma we get from the assumption that reasons to suspend always trump reasons to believe.

${ }^{5}$ As we will see below, in the case where $S$ has conclusive evidence for $p$, but also has reasons to consider $p$ that are perfectly counterbalanced by reasons to ignore $p$, BALANCE will say that believing $p$ and taking no attitude toward $p$ are both permissible, while withholding with respect to $p$ and believing $\neg p$ are both impermissible. 
gradual. What happens as you move from the top of the chart to the bottom, as the letters become increasingly hard to see?

Let ' $\mathcal{L}$ ' range over the 26 letters of the alphabet. Say that a letter on the chart is transparent just in case, for some value of ' $\mathcal{L}$,' you are rationally required to believe that it's an $\mathcal{L}$; say that a letter on the chart is opaque just in case, for some value of ' $\mathcal{L}$,' you are rationally required to withhold with respect to the proposition that it's an $\mathcal{L}$; and say that a letter on the chart is translucent just in case, for some value of ' $\mathcal{L}$,' you are rationally permitted to believe that it's an $\mathcal{L}$, but not rationally required to believe that it's an $\mathcal{L}$, since you are also rationally permitted to withhold with respect to the proposition that it's an $\mathcal{L}$. The letters at the bottom of the chart are all (we can suppose) opaque while the large ' $\mathrm{E}$ ' at the top is transparent. But what about the letters in the middle? Are they all either opaque or transparent? The letters get easier to see as you move from the bottom of the chart to the top. Is it plausible that, as you move from the bottom to the top, the first transparent letters follow immediately after the last opaque letters? This would be plausible if the letters on each line were much larger than the letters on the line below them. But we can suppose that they aren't. What should we say about the letters in the middle of the chart, then, supposing that the transition from the opaque letters at the bottom to the transparent letters at the top is very gradual?

The obvious thing to say, it seems to me, is that at least some of the letters in the middle of the chart are translucent. I find this conclusion just as plausible as the claim that, if your reasons for performing some action equal your reasons for not performing that action, you will be rationally permitted to perform that action, but not rationally required to perform it, since refraining from performing that action will also be rationally permissible. Imagine an archery competition. At each station you can shoot or refrain from shooting. Some of the targets are very close. You can't miss them. Other targets are very far. You can hardly see them, much less hit them. The rest of the targets are various distances in between. You gain a point for every target you hit, you lose a point for every target you miss, and you neither gain nor lose a point when you refrain from shooting at a target. We can easily fill in the details of this case so that, at some of the stations, you are rationally permitted to shoot, but not rationally required to shoot, since refraining would also be rationally permissible. But the same seems true of the eye chart. At the top of the chart, your evidence demands belief, and thus supports belief over suspension. At the bottom of the chart, your evidence demands suspension, and thus supports suspension over belief. Somewhere in the middle, (surely) your evidence supports belief and suspension equally well. But in this case, don't we have a translucent letter? This question is important because, if 
there is a translucent letter on the chart, then there are cases where one is rationally permitted to take multiple doxastic attitudes toward a proposition. Reflecting on cases like the eye chart case, it seems plausible that one might be rationally permitted to take multiple doxastic attitudes toward a proposition, since it seems plausible that one's evidence might support belief and suspension equally well. The fact that BALANCE entails that one may be rationally permitted to take multiple doxastic attitudes toward a proposition is thus arguably a virtue of the view, not a vice. ${ }^{6}$

\section{Rational Inattention}

Cohen's third and final objection to BALANCE is that it will often get the wrong result in cases where someone is simply not considering a proposition. There are only three doxastic attitudes that $S$ might take toward a proposition: she can believe it, she can believe its negation, and she can suspend. According to BALANCE, $S$ is rationally required to believe $p$ if her reasons to believe $p$ are stronger than her reasons to not believe $p$, and she is rationally required to suspend on $p$ if her reasons to suspend on $p$ are stronger than her reasons to not suspend on $p$. As Cohen points out, however, $S$ doesn't take any attitude towards $p$ if she never considers $p$. And this is a problem, says Cohen, because $S$ is not rationally required to take any attitude toward $p$ unless she $i$ s considering $p$. As he puts it,

the permitted/required distinction looks very different for belief than it does for action. In the case of action, the distinction hinges on the relative strength of one's reasons to $\operatorname{do} A$ and not to do $A$-between having sufficient reasons and having conclusive reasons. In the case of belief, the distinction between requirement and permission is not a distinction between sufficient and conclusive reasons. One is rationally permitted to believe $p$ just in case one's reasons permit one to

${ }^{6}$ Objection: This argument fails to distinguish between being rationally required to do something and knowing that one is rationally required to do it. On the right way to think about the eye chart, every letter is either transparent or opaque. There are no translucent letters. The letters in the middle seem translucent, of course, but this is only because, for each letter in the middle, it's impossible to know whether that letter is transparent or opaque. Reply: This objection seems as about as plausible as the claim that there's no such color as grey, that every color in the image below is either black or white, and that the colors in the middle only seem grey because, for each color in the middle, it's impossible to know whether it's black or white.

Obviously, I don't find this claim plausible. 
believe $p$. Whether one is required to believe is not a further fact about one's reasons, i.e., there is no such thing as having conclusive reasons to believe (where having conclusive reasons entails being required to believe). Being rationally required to believe is not determined by the reasons you have to believe. Rather it involves facts about one's psychology. (p. 10)

According to Cohen, the relevant fact about one's psychology is whether one is attending to the question whether $p$. Since he thinks that rationality will never permit more than one doxastic attitude toward $p$, he concludes that "one is required to adopt a doxastic attitude toward $p$ just in case one is permitted to believe $p$ and one is attending to whether $p$ " (ibid). According BALANCE, however, there is such a thing as having conclusive reasons to believe, since $S$ is rationally required to believe $p$ if her reasons to believe $p$ are stronger than her reasons to not believe $p$.

I agree with Cohen that $S$ does not take any attitude towards $p$ if she never considers $p$. I am not yet convinced, however, that there is no such thing as having conclusive reasons to believe. Suppose I take my son to the park. I read a book while he plays. When I look up I see a gun in his hand. Not a toy, but a real handgun. Someone left it at the park, my son found it, and now he's now playing with it, oblivious to the danger. If I never attend to the question whether the gun is loaded, I will have no doxastic attitude toward that proposition. Does it follow that I am not rationally required to have any doxastic attitude toward that proposition? I want to say, 'no!' I had better consider the question whether the gun is loaded, and then either believe that it is loaded, believe that it isn't loaded, or suspend judgement on the question whether it's loaded, depending on my evidence. If it's clear that the gun is loaded, then the combination of my evidence that the gun is loaded and my reasons for considering the question whether the gun is loaded gives me conclusive reason to believe that the gun is loaded. This, I think, is intuitively the right thing to say. But if this is the right thing to say, then it's false that there is no such thing as conclusive reason to believe.

With respect to BALANCE and the more general question whether reasons to believe differ importantly from reasons to act, I am unconvinced that there is anything very interesting about the fact that, in addition to believing $p$, believing $\neg p$, and withholding with respect to $p, S$ can also take no attitude toward $p$. Notice that choices between actions can have a similar structure. Suppose you're sitting in the shade and a social scientist asks you to participate in an experiment. Participation involves taking a test. This test contains one question followed by three boxes marked 'T,' 'F,' and 'W.' You answer the question by checking exactly one of these boxes. There are consequences for your choice between 'T,' 'F,' and 'W,' depending on the 
truth-value of the proposition that you are asked about. If the proposition is true, checking the box marked ' $\mathrm{T}$ ' will have the best consequences, checking the box marked 'W' will have the second best consequences, and checking the box marked ' $F$ ' will have the worst consequences. However, if the proposition is false, things go the other way around; checking the box marked ' $\mathrm{T}$ ' will have the worst consequences, checking the box marked ' $\mathrm{W}$ ' will have the second worst consequences, and checking the box marked ' $\mathrm{F}$ ' will have the best consequences. You look at the question and see that it concerns $p$. What should you do? Should you check the box marked 'T,' check the box marked ' $F$ ', check the box marked 'W'-or check no box at all, which is tantamount to not participating in the experiment? What does BALANCE say you should do?

BALANCE says basically the same thing about this case as it says about any case of belief. According to BALANCE, you are rationally required to check the box marked ' $\mathrm{T}$ ' (for example) just in case your reasons to check the box marked ' $\mathrm{T}$ ' outweigh your reasons to not check the box marked 'T.' 'There are three ways to not check the box marked 'T:' you can check the box marked 'F,' you can check the box marked 'W,' and you can not participate in the experiment. Thus, according to BALANCE, you are rationally required to check the box marked ' $\mathrm{T}$ ' just in case (a) your reasons to check the box marked ' $T$ ' outweigh your reasons to check the box marked ' $F$,' (b) your reasons to check the box marked ' $\mathrm{T}$ ' outweigh your reasons to check the box marked 'W', and (c) your reasons to check the box marked ' $\mathrm{T}$ ' outweigh your reasons to not participate in the experiment. This is point-for-point analogous to the implications of BALANCE for believing $p$. According to BALANCE, you are rationally required to believe $p$ just in case (a) your reasons to believe $p$ outweigh your reasons to believe $\neg p$, (b) your reasons to believe $p$ outweigh your reasons to withhold with respect to $p$, and (c) your reasons to believe $p$ outweigh your reasons to not consider the question whether $p$.

With respect to BALANCE and the more general question whether there are important differences between reasons to believe and reasons to act, not considering a question seems perfectly analogous to not participating in the social scientist's experiment. If cases where someone is not considering a question give us counterexamples to BALANCE, it seems that we should get analogous counterexamples from cases where you don't participate in an experiment like this. I doubt that BALANCE will have any trouble handling cases where you don't participate in an experiment like this, so I doubt that cases where someone is not considering a question give us counterexamples to BALANCE. But the more important point is that, 
if BALANCE performs equally well on cases where someone is not considering a question and cases where someone does not participate in an experiment like this, then we have just discovered a similarity between reasons to believe and reasons to act. This is important because Cohen isn't just arguing against BALANCE; he's arguing that, because BALANCE gets the wrong results when applied to belief but not when applied to action, there are important differences between reasons to believe and reasons to act. So far as I can tell, the fact that $S$ takes no doxastic attitude toward a proposition unless she considers it does not show that there are important differences between reasons to believe and reasons to act. ${ }^{7}$

\section{Transparency, Reasons to Believe, and Reasons to Act}

Are there are important differences between reasons to believe and reasons to act, then? If I were going to answer 'yes,' I would take a page from Richard Moran (2001), Jonathan Adler (2002), Nishi Shah (2003), Shah and Velleman (2005), and others, and argue like this:

The question whether I should believe $p$ is transparent to the question whether $p$ is true. That is, the deliberative question whether I should believe $p$ gives way to the factual question whether $p$ is true. To answer the factual question whether $p$ is true, however, I must focus entirely on my evidence for and against $p$. After all, nothing else is relevant to the truth of $p$. This means that the question whether I should believe $p$ depends entirely on my evidence. The question whether I should do $A$ does not depend entirely on my evidence, however. (Among other things, it also depends on my values-if not the values I do have, then at least on the values that I should have.) Reasons to believe and reasons to act differ in at least this respect, then: the former depend entirely on evidence while the latter do not.

The first premise of this argument (what people usually call 'transparency') is standardly taken as a datum, and this argument seems pretty plausible downstream from this premise. I suspect, however, that transparency is false.

Suppose you and I have exactly the same evidence for $p$ but we disagree about whether we should believe $p$ or withhold. Suppose further that our disagreement

\footnotetext{
${ }^{7}$ On page 8, Cohen says this: “if only one attitude is permitted, isn't that attitude required? The analogous point is true for action. If only one act is permitted, then that act is required. The reason for this is that you have to do something. You can only not do $A$ by doing something else. Belief is different." But is it really true that you can only not do $A$ by doing something else? Can't you not do $A$ by not doing anything at all?
} 
stems entirely from a disagreement about epistemology. We disagree about the minimum amount of evidence required for justified or rational belief. We agree that we have exactly evidence $e$ for $p$, we agree that the probability of $p$ on $e$ is exactly $n$ (for some value of ' $n$ ' $<1$ ), and so on; it's just that you think $e$ is sufficient evidence for rational or justified belief in $p$ while I think the threshold is higher. Both of us realize that we don't know exactly where the threshold lies. In this case, can either of us answer the question whether we should believe $p$ by focusing entirely on our evidence? Arguably, 'no.' Consider an analogy. Suppose we agree that we have exactly ten gallons of gas left in our tank, and we also agree that our car gets exactly 35 miles per gallon, but we disagree about whether we should stop to get more gas because we disagree about the distance to our destination. Attempting to answer the question whether we should believe $p$ by focusing entirely on our evidence seems analogous to attempting to answer the question whether we should stop to get more gas by focusing entirely on the amount already in our tank. Arguably, just as the amount of gas in our tank cannot, all by itself, settle the question whether we should get more gas, our evidence for $p$ cannot, all by itself, settle the question whether we should believe $p$. To answer that question, we must do some epistemology. We must answer the question (to some satisfactory degree of precision) how much evidence is enough evidence for rational or justified belief. ${ }^{8}$ But for many values of ' $p$,' this question will be completely irrelevant to the factual question whether $p$ is true.

Why do we theorize about rationality, justification, and other epistemic statuses? Epistemology exists, I suspect, because the deliberative question whether I should believe $p$ does not always give way to the factual question whether $p$ is true. Or, at least, I suspect this is at least part of the explanation why epistemology exists. But if so, then epistemology exists at least in part because transparency is false. Transparency, however, is the basis of what I think is the best argument for an important difference between reasons to believe and reasons to act. This leaves me unsure whether there are important differences between reasons to believe and reasons to act.

I agree with a lot in "Reasons to Believe and Reasons to Act." Cohen spends most of the paper arguing that Schroeder's cases involving practical interests, forthcoming evidence, and the mental resources required for forming a belief do not provide clear examples of non-evidential reasons to withhold, and arguing that, even if they did,

\footnotetext{
8 And notice that the answer to this question may turn on the values of true belief, false belief, and no belief, respectively. This might be the case even if these values are not affected by changes in one's practical circumstances. See, for example, Riggs (2003).
} 
these reasons would often be inoperative. I agree with Cohen on both of these points. But Cohen draws two conclusions from these points that I am not sure follow: that BALANCE runs into trouble when applied to belief, and that there are important differences between reasons to believe and reasons to act. To me, it still seems an open question whether BALANCE is plausible when applied to belief, and whether there are important differences between reasons to believe and reasons to act.

\section{References}

Adler, Jonathan (2002), Belief's Own Ethics (Cambridge, MA: MIT Press).

Moran, Richard (2001), Authority and Estrangement: An Essay on Self Knowledge (Princeton: Princeton University Press).

Riggs, Wayne (2003), “Balancing Our Epistemic Goals,” Nous, Vol. 37, No. 2: pp. 342-52.

Schroeder, Mark (2012), "Stakes, Withholding, and Pragmatic Encroachment on Knowledge," Philosophical Studies, Vol. 160: pp. 265-85.

Shah, Nishi (2003), "How Truth Governs Belief," The Philosophical Review, Vol. 112, No. 4: pp. 447-82.

(2005), “A New Argument for Evidentialism," The Philosophical Quarterly, Vol. 56, No. 225: pp. 481-98.

Shah, Nishi and David Velleman (2005), "Doxastic Deliberation," The Philosophical Review, Vol. 114, No. 4: pp. 497-534. 